\title{
Une élite transnationale : la fabrique d'une identité professionnelle chez les fonctionnaires du Haut Commissariat des Nations Unies aux Réfugiés
}

\author{
A Transnational Elite: The Making of a Professional Identity among the \\ International Staff of the United Nations High Commissioner for Refugees \\ Una élite transnacional: la fábrica de una identidad profesional de los \\ funcionarios internacionales del HCR
}

\section{Marion Fresia}

\section{OpenEdition}

\section{Journals}

Édition électronique

URL : https://journals.openedition.org/remi/4999

DOI : $10.4000 /$ remi.4999

ISSN : $1777-5418$

Éditeur

Université de Poitiers

Édition imprimée

Date de publication : 1 décembre 2009

Pagination : 167-190

ISBN : 978-2-911627-53-8

ISSN : 0765-0752

\section{Référence électronique}

Marion Fresia, « Une élite transnationale : la fabrique d'une identité professionnelle chez les fonctionnaires du Haut Commissariat des Nations Unies aux Réfugiés », Revue européenne des migrations internationales [En ligne], vol. 25 - n³ | 2009, mis en ligne le 01 décembre 2012, consulté le 14 avril 2022. URL : http://journals.openedition.org/remi/4999; DOI : https://doi.org/10.4000/remi. 4999 


\title{
Une élite transnationale : la fabrique d'une identité professionnelle chez les fonctionnaires du Haut Commissariat des Nations Unies aux Réfugiés
}

\author{
Marion FRESIA*
}

Au-delà de la seule compréhension des mouvements migratoires, l'anthropologie s'intéresse de plus en plus aux institutions investies dans le champ de la migration et de l'asile'. Depuis la fin des années quatre-vingt, une réflexion conséquente s'est notamment développée sur le Haut Commissariat des Nations Unies aux Réfugiés (HCR). Une première approche a consisté à interroger les catégories juridiques et les soubassements idéologiques sur lesquels le HCR légitime son mandat, et à étudier la portée de son action au regard de ses objectifs officiels. Dans cette perspective, de nombreux travaux ont dénoncé les effets pervers des interventions du HCR et montré comment, loin d'atteindre sa mission de protection de droits des réfugiés, il participe, en pratique, à servir les intérêts des États en renforçant leur contrôle sur les mouvements de population ${ }^{2}$. Souhaitant avant tout mettre à jour les mécanismes de la domination liés aux interventions du HCR, ces analyses ont généralement considéré le HCR comme un acteur homogène, doté d'une rationalité et d'une intentionnalité propres. Une deuxième approche a consisté à s'intéresser au HCR en tant qu'institution complexe, en initiant une réflexion sur son histoire (Loescher, 2001), ses modes de gouvernance (Valluy, 2007 et 2008) ou encore sa culture organisationnelle (Wigley, 2005). Cette perspective reste néanmoins minoritaire chez les anthropologues, que ce soit pour des raisons d'accessibilité ou par un certain penchant à accorder un intérêt plus grand aux groupes dominés qu'aux classes dominantes. Ainsi, on sait encore peu de choses sur la « machinerie interne » du HCR, ses lieux informels du pouvoir, les motivations et les trajectoires professionnelles de ses membres ; l'univers de

\footnotetext{
* Professeure assistante, Institut d'ethnologie/Maison d'analyse des processus sociaux, 4 rue SaintNicolas, 2000 Neuchâtel, SUISSE ; marion.fresia@unine.ch

1 Je tiens à remercier Denys Cuche, Karen Akoka, Ellen Hertz et Giulia Scalettaris pour leurs précieux commentaires et suggestions.

2 Pour une revue plus compréhensive de la littérature sur les réfugiés et l'action du HCR en sciences sociales, voir Backewell (2007), Fresia (2007) et Foxen (2009).
} 
sens dans lequel ils évoluent; les conflits d'intérêts ou de représentations qui les opposent - autant d'éléments qui influencent pourtant la manière dont l'organisation fabrique des normes à prétention universelles dans le champ de l'asile et de la migration. De récentes recherches anthropologiques prenant spécifiquement pour objet d'étude les organisations internationales attestent pourtant de la portée d'un regard qui part de l'intérieur (Mosse, 2005 ; Anders, 2005 ; Dembour et Kelly, 2007 ; Atlani-Duault, 2005 ; Harper, 1998 ; Muller, 2009 ; Hertz, à paraître $\left.{ }^{3}\right)$. Ces travaux montrent comment l'anthropologie - à travers une approche au " ras le sol », une micro-échelle d'observation ou encore la reconstruction de réseaux professionnels - peut aider à mieux saisir la complexité des processus sociaux sous-jacents à la production de politiques publiques internationales et aux dynamiques internes à ces institutions. Ils en dévoilent l'hétérogénéité, la matérialité mais aussi la diversité des rationalités à l'œuvre (Abelès, 1995) et démontrent comment en prenant au sérieux le cadre d'action et de réflexion de leurs membres et la vision critique qu'ils développent sur leurs propres pratiques, le discours scientifique sur les élites internationales et plus largement sur la « gouvernance globale » peut devenir plus nuancé et moins dénonciateur (Lavigne Delville, 2007 ; Baré, 2006).

S'inscrivant dans cette dernière approche, et plus largement dans une perspective d'anthropologie des organisations (Nader, 1972 ; Wright, 1994 ; Czarniawska, 1992 ; Abelès, 1995 ; Bellier, 1997), cet article se propose d'explorer l'univers social des acteurs cosmopolites qui constituent le HCR. Il se basera sur des analyses tirées de deux années de participation-observante au sein de cette organisation (2005-2007), où j'ai occupé un poste de fonctionnaire internationale à Genève ${ }^{4}$, et sur un ensemble d'entretiens menés $a$ posteriori avec d'anciens collègues ${ }^{5}$. De cette expérience, un premier constat s'impose : envisager le HCR comme un seul et même acteur, doté d'une intentionnalité propre, semble impossible. Administration publique intergouvernementale comprenant 72 Étatsmembres, l'agence onusienne ne compte pas moins de 6000 employés d'une soixantaine de nationalités différentes, et 263 bureaux locaux répartis dans 116 pays. Elle est constituée à la fois de juristes ( Protection officer ») chargés de négocier au plus haut niveau des normes internationales sur l'asile et d'encourager les gouvernements à les respecter, et d'humanitaires («Programme officer») déployés au cœur de zones en crise pour accueillir des réfugiés, aménager des camps et coordonner des programmes d'aide ${ }^{6}$. Soumis à un

3 Un réseau européen pour l'étude des organisations internationales s'est constitué en 2007 à l'initiative du Laboratoire d'Anthropologie des Institutions et des Organisations sociales (CNRS/ EHESS).

4 Basée au niveau du département des opérations au siège de l'organisation, j'occupais une fonction d'appui technique au terrain dans le domaine de l'éducation. Dans ce cadre, j'ai été amenée à effectuer plusieurs missions de formation et d'évaluation dans une dizaine de pays d'Afrique sub-saharienne où le HCR est implanté.

5 Pour des raisons de confidentialité, les personnes citées ne seront pas nommées de même que leur fonction et leur lieu d'affectation. 18 entretiens formels et enregistrés ont, à ce jour, été menés avec des fonctionnaires internationaux du HCR ainsi que 8 entretiens avec des délégués des Étatsmembres du HCR.

6 La distinction opérée entre juristes et humanitaires est celle de l'auteure. D'un point de vue émic, elle s'exprime à travers l'opposition entre les Protection Officer, spécialisés dans la protection juridique, et les Programme Officer, spécialisés dans l'assistance humanitaire (Programme officer). 
système de rotation de postes, ces juristes et ces humanitaires circulent, de plus, en permanence, changeant d'affectations géographiques et de fonctions tous les deux à cinq ans. Aux côtés de cette élite transnationale, appelée dans le jargon du HCR «staff international » ou «professional staff », opère par ailleurs le personnel national, surnommé « staff national » ou « general staff », recruté localement et effectuant, en principe, des tâches administratives?. Les uns comme les autres sont placés sous l'autorité du "senior staff committee », qui forme la direction du HCR. Ce dont on parle ici n'est donc pas une organisation en tant qu'entité homogène et fermée, mais une véritable nébuleuse bureaucratique et humaine aux contours flous, construite sur la mobilité ininterrompue d'une partie de ses membres et traversée par de multiples conflits entre divers niveaux institutionnels (Genève vs le terrain), cultures professionnelles (juridique, humanitaire et administrative) et catégories de personnel (staff international ; staff national mais aussi senior management). Ses frontières sont d'autant plus poreuses que certains individus occupant une position a priori externes à l'organisation, tels que les membres de la « société civile » et du milieu universitaire, participent aussi pleinement à la production des politiques du HCR via divers réseaux d'experts. À l'instar d'autres organisations internationales, le HCR ne peut donc pas être appréhendé comme une totalité finie mais apparaît plutôt comme un dispositif mouvant (Muller, 2009 : 28).

Qu'une agence internationale soit dynamique, hétérogène et conflictuelle n'est toutefois pas ce qui m'a le plus surprise au cours de mes deux années d'immersion au sein du HCR. Mon étonnement est plutôt venu de la capacité de cette institution à produire un certain semblant d'homogénéité, de cohésion et d'ordre, lui permettant de légitimer son action, de survivre et de se reproduire. À l'instar d'autres anthropologues qui ont travaillé sur la Banque Mondiale (Mosse, 2005) et la FAO (Muller, 2009), j'ai par exemple été interpellée par la manière dont les membres du HCR construisent des politiques et des récits " ordonnés » permettant de conférer du sens à la multiplicité et l'hétérogénéité de pratiques qui relèvent le plus souvent, de processus de bricolage et d'improvisation. Mais j'ai aussi été frappée par la manière dont ils arrivaient à définir et redéfinir constamment des cadres de référence et des repères identitaires communs, leur permettant de communiquer, de travailler et d'agir ensemble par-delà la pluralité de leurs cultures, de leurs langues et de leurs origines. Ceci était plus particulièrement étonnant chez les fonctionnaires internationaux : malgré la diversité de leurs nationalités, de leurs contrats (permanents ou temporaires), de leurs fonctions (juristes ou humanitaires) et au-delà des rapports de compétition qui les opposent, ils semblaient relier par les mêmes manières de dire, de faire et de se représenter leur institution, et partager la même conviction d'appartenir à une même « famille».

Dans cet article, je souhaite approfondir ce dernier constat en explorant certains des éléments - cognitifs, rituels, idéologiques ou pratiques - qui constituent le socle de

7 L'opposition sémantique « general vs. professionnal staff» reflète l'idée que les seconds disposent d'une expertise et d'un savoir spécifiques alors que les premiers resteraient de simples administrateurs. L'observation des pratiques quotidiennes permet néanmoins de constater que cette frontière est sans cesse renégociée. Le staff national est fréquemment amené à exercer des tâches de spécialistes du fait de sa présence effective et permanente sur le terrain, alors que le staff international, soumis au système de rotation, dispose souvent d'une vision plus globale des interventions du HCR et exerce essentiellement des tâches de coordination. 
cet univers de sens partagé et qui participent à fabriquer un sentiment d'appartenance collective entre des acteurs sociaux hétérogènes et extrêmement mobiles. Ma réflexion se basera uniquement sur les fonctionnaires internationaux du HCR soumis au système de rotation (international staff), n'occupant pas de postes de direction. Elle s'organisera autour de l'analyse de quelques catégories emic, omniprésentes dans les discours qu'ils tiennent sur leur organisation et sur eux-mêmes, quels que soient leur degré d'ancienneté et leur origine nationale : des notions telles que « le mandat», le « terrain », la « maison» et le « siège » nous serviront ainsi de porte d'entrée pour étudier comment cette élite transnationale construit, reconstruit et mobilise continuellement une identité professionnelle, à travers (i) la défense d'une cause commune (ii) des formes de socialisation et d'apprentissage fortement ritualisées (iii) des modes de vie et des repères spatio-temporels similaires (iv) et le partage d'un ensemble de frustrations vis-à-vis de l'institution. Cette analyse m'amènera également à ouvrir, de manière transversale, diverses pistes de réflexion sur les inévitables décalages qui s'opèrent entre représentations idéalisées de l'institution et expériences vécues dans l'exercice quotidien de son métier.

\section{« LE MANDAT DE PROTECTION » : LA DÉFENSE D’UNE CAUSE COMMUNE}

« Notre plus grande force, c'est notre mandat qui est inscrit dans les textes. Personne ne conteste que le HCR est l'agence de protection des réfugiés. C'est nous qui avons le mandat de protéger et d'assister les réfugiés mais aussi d'assurer que des solutions durables soient trouvées à leur situation $»^{8}$.

Lorsque l'on demande au staff international du HCR quelle est la plus grande force de son institution, la réponse la plus souvent obtenue se tient en un mot : « le mandat». Ce terme fait référence au mandat de « protection internationale » que l'agence onusienne exerce sur une catégorie de population bien définie juridiquement : « les réfugiés ». Il est omniprésent dans les discours et rapports officiels de l'agence onusienne mais aussi dans le langage quotidien et informel du personnel. Toutes les activités menées par les différents départements du HCR, au siège comme sur le terrain, se légitiment en son nom. La référence au mandat exprime en premier lieu la conviction largement partagée par l'ensemble du staff que les réfugiés constituent un problème à l'échelle internationale, auquel il s'agit d'apporter des solutions. Tous les fonctionnaires internationaux que nous avons côtoyés et interviewés adhérent ainsi sans ambiguiité à la mission du HCR telle qu'elle est formulée dans ses statuts : « le HCR (...) assume les fonctions de protection internationale (...) et de recherche de solutions permanentes au problème des réfugiés (...) en aidant les gouvernements (...) à faciliter le rapatriement librement consenti de ces réfugiés ou leur assimilation dans de nouvelles communautés nationales $»^{9}$. La plupart croit également fermement aux principes de la Convention de Genève relative au statut de réfugié (1951), qui édicte les droits fondamentaux de celui-ci et qui le définit comme toute personne ayant une crainte fondée de persécution dans son pays d'origine et ne pouvant plus se réclamer

8 Une fonctionnaire internationale (34 ans), travaillant pour le HCR depuis 2003, rencontrée à Genève en 2009.

9 Statuts du HCR, art. 1, 1950.

REMI 2009 (25) 3 pp. 167-190 
de la protection de ce pays (article 1). Les notions de « protection » et de "réfugiés », associées à celle de mandat, sont donc au cœur de l'univers de sens des fonctionnaires. Elles véhiculent une vision du monde commune, qui s'inscrit dans un double héritage : celui de la philosophie des lumières d'une part, qui s'insurge contre les injustices perpétrées par des gouvernements tyranniques ; et celui des nationalismes modernes de l'autre, qui véhiculent une conception territoriale et souveraine des identités (Malkki, 1995). Dans cette perspective, la mobilité, la persécution et l'absence de protection juridique d'un État sont perçues comme « hors normes » et la normalisation de cette situation, qui doit passer par la réintégration du réfugié dans son pays d'origine ou à défaut d'un autre pays, est appréhendée comme une cause « juste » qu'il s'agit de défendre. Toute tentative de sortir de cette vision du monde - en remettant en cause, par exemple, la définition juridique du réfugié - se heurte à de fortes résistances voir même à l'impossibilité, pour certains, d'envisager d'autres alternatives possibles.

Les fonctionnaires internationaux du HCR se rejoignent ainsi en premier lieu au sein d'un même cadre cognitif, calqué sur une grille de lecture juridique et droits de l'hommiste du monde, qui leur permet d'interagir et de se comprendre au quotidien. Une bonne partie d'entre eux est, de fait, issue d'une formation juridique (droit international des droits de l'homme, droit des administrations internationales, ou sciences politiques) à laquelle s'ajoute souvent une expérience préalable dans le domaine social (bénévolat). Quelle que soit leur origine ou leur fonction, tous mobilisent ainsi jour après jour leurs savoirs pour jouer un rôle dans l'élaboration de solutions à ce qu'ils considèrent être une cause juste et légitime. Sur la base de ce premier constat, on peut dès lors avancer qu'ils forment une même " communauté épistémique ». De plus en plus mobilisée dans le champ des sciences sociales, celle-ci se définit comme des "réseaux d'experts qui ont en commun un modèle de causalité et un ensemble de valeurs politiques. Ils sont unis par la croyance forte en ce modèle et dans l'engagement de traduire cette croyance en politique publique et que ce faisant le bien-être de l'humanité en sera amélioré » (Haas, 1990 : 42). Cette croyance est construite comme allant de soi : elle est fondée sur l'adhésion à une morale qui se veut universelle et incontestable, celle de la défense des droits de l'homme en général et des réfugiés en particulier - une morale qui élude donc la dimension historiquement située et idéologiquement orientée du droit des réfugiés et du processus de construction de cette catégorie comme objet de politiques publiques internationales ${ }^{10}$.

Si elle révèle une conviction forte d'agir pour le bien de l'humanité, la référence systématique au mandat permet aussi de se distinguer face aux autres institutions internationales. Dans la plupart des discours recueillis, elle est en effet avant tout mobilisée pour souligner la spécificité du HCR par rapport à d'autres institutions onusiennes et humanitaires avec lesquelles il entre directement en concurrence sur le marché de l'humanitaire : « notre mandat nous donne du pouvoir par rapport aux autres agences de l'ONU; nous avons une autorité reconnue pour tout ce qui concerne les problèmes des réfugiés, nous

10 Rappelons que ce processus remonte seulement à l'entre-deux-guerres, dans un contexte marqué par l'affirmation de l'hégémonie des États-nations et l'introduction de politiques d'immigration, de contrôle des frontières et de classification administrative des individus (Loescher, 2008 : 7). Pour la première fois, les personnes fuyant leur zone d'origine et démunies de toute pièce d'identité furent alors perçues comme un « problème » par les acteurs étatiques et des réseaux d'experts se constituèrent pour y apporter des solutions à prétention universelle. 
sommes les seuls à avoir un objectif clair alors que les autres ont des champs de compétence qui s'empiètent les uns sur les autres $\gg{ }^{11}$. Le HCR est, de fait, la seule agence de l'ONU à s'être vue confiée par l'Assemblée générale des Nations Unies un droit de regard exclusif sur une catégorie de personnes dont les contours sont définis dans une convention internationale. Cette légitimité juridique, reconnue à l'échelle internationale, confère à ses membres le sentiment de détenir une « autorité morale » exclusive mais aussi universelle sur tout ce qui est lié à l'asile. Elle permet à la fois d'affirmer son identité professionnelle face aux autres agences de l'ONU mais aussi de revendiquer ce qu'il est bon et juste de faire dans un certain domaine de la société et même de «définir les catégories dans lesquelles ce domaine peut être pensé » (Hugues, 1996 : 100). Aux convictions morales qui fondent la base de cette communauté épistémique se mêlent ainsi en même temps, des intérêts proprement institutionnels, défendus par des réseaux d'acteurs qui cherchent à conserver leur contrôle sur la définition du champ d'expertise qu'ils ont développé. De fait, on remarque qu'en conservant le contrôle de la définition de la notion de «protection », le HCR a réussi au fil des années à élargir son champ de compétences et à s'adapter à l'évolution des demandes de ses « clients », ces derniers n'étant pas les réfugiés mais les États, qui financent à $90 \%$ son activité sous forme de contributions volontaires. Ainsi, le mandat de l'organisation s'est d'un côté élargi à différentes catégories de victimes que les seuls réfugiés et de l'autre, à de nombreux autres domaines que la seule protection juridique $^{12}$. Depuis le début des années quatre-vingt-dix en particulier, avec la systématisation des camps comme modalités d'assistance aux réfugiés et la multiplication des partenariats avec des ONGs de plus en plus nombreuses sur le terrain, le volet humanitaire des interventions du HCR est devenu bien plus important que le volet juridique, ce qui s'est traduit par une explosion de la taille et du budget de l'organisation ${ }^{13}$. La légitimation de ce processus s'est opérée en sortant d'une définition restreinte de la "protection ", basée sur les seuls droits des réfugiés édictés par la Convention de Genève, et en défendant une acceptation plus large de cette notion, incorporant les avancées du droit international des droits de l'homme et du droit international humanitaire. Comme le souligne un fonctionnaire : «le mandat de protection n'a finalement jamais été exécuté tel qu'il est formulé dans les statuts $d u H C R »{ }^{14}$ : différentes interprétations lui ont été données en fonction des époques historiques mais aussi des rapports de force internes à l'institution (Loescher, 2001). Si l'institution a pendant longtemps été dominée par les juristes, on a ainsi assisté à une montée en puissance des humanitaires depuis le tournant des années quatre-vingt, quatre-vingt-dix ${ }^{15}$. C'est pourquoi le contenu de sens attribué à la notion de "protection» inclut désormais une dimension matérielle (droit à la vie, qui se décline en droit à l'assis-

11 Un fonctionnaire international (49 ans), travaillant pour le HCR depuis 1994, rencontré à Genève en 2009.

12 Sur l'évolution du mandat du HCR et la multiplication des catégories de victimes, voir Loescher et al., 2008.

13 En 1951, le HCR comptait 34 personnes basées principalement à Genève avec un budget de 300000 dollars. Aujourd'hui, il compte 6500 personnes pour un budget de plus d'un milliard de dollars (Loescher, 2001 : 78).

14 Un fonctionnaire international (45 ans), travaillant pour le HCR depuis 1996, rencontré à Genève en 2008.

15 Et ceci malgré la tentative du Haut commissaire Lubbers de recentrer le HCR sur ses activités de protection juridique au début des années deux mille. 
tance alimentaire et sanitaire) en plus de sa dimension strictement juridique et politique (droit d'asile, qui se décline en respect des procédures d'asile et des libertés individuelles).

Aujourd'hui, le HCR reste toutefois traversé par une tension structurante entre les juristes, soucieux de préserver en premier lieu le respect du droit d'asile et de limiter le mandat du HCR à sa fonction de garant de la Convention de Genève, et les humanitaires, souhaitant avant tout répondre aux besoins matériels immédiats (abris, nourriture, santé) des victimes qu'ils côtoient sur le terrain au nom du droit humanitaire (Loescher et al., 2008 : 78). Les premiers sont plus enclins à confronter les États pour qu'ils respectent leurs obligations internationales en matière d'asile, et tendent à considérer qu'un élargissement trop grand des activités du HCR et des catégories de personnes à protéger risquerait de se faire au détriment de la sauvegarde du droit des réfugiés : « en voulant s'engager dans des domaines humanitaires qui ne sont pas de nos compétences, non seulement on ne fait pas bien notre travail mais en plus on le fait au détriment de notre mandat principal. $\dot{A}$ $X$ par exemple, on nourrissait des déplacés internes pendant que des réfugiés se faisaient refouler aux frontières... De même qu'aujourd'hui, on investit le champ de la migration alors que le droit d'asile est en pleine crise $»^{16}$. Tandis que les seconds sont prêts à faire plus de concessions sur le droit d'asile face aux États, pour s'assurer, en retour, un accès plus libre aux personnes vulnérables à assister. Ils font preuve de plus de pragmatisme et ont une interprétation plus extensible du mandat de protection du HCR : " on est toujours en train de balbutier quand il faut en sortir de notre mandat alors que sur le terrain, on a une capacité d'intervention qui pourrait nous permettre de prendre beaucoup plus de responsabilités pour des personnes qui en ont besoin et dont on peut sauver la vie ${ }^{17}$. Cette tension resurgit à chaque fois que le HCR doit se repositionner sur l'arène internationale en fonction de l'évolution des enjeux et des préoccupations politiques de ses principaux bailleurs de fonds. Si elle partage un même cadre cognitif, une communauté épistémique ne constitue donc pas pour autant un ensemble homogène : "le savoir expert lui-même n'est pas réductible à une parole consensuelle » (Kott, $2008: 26)$. Une institution doit être envisagée comme un processus continu et quotidien de négociation autour du « sens » attribué à sa mission (Strauss et al., 1963) et comme le produit d'une confrontation entre diverses représentations (Abelès, 1995). Ces tensions restent néanmoins toujours confinées au sein d'un même cadre d'interprétation plus large, qui se définit à travers le prisme du droit international. On peut ainsi penser que les fonctionnaires du HCR construisent leur communauté épistémique sur un « ordre sans cesse négocié » (Strauss et al, 1963), qui se structure autour d'un dilemme récurrent lié à la difficulté de concilier la sauvegarde de droits politiques (droit à l'asile) avec celle du droit humanitaire (droit à la vie).

\section{«LE TERRAIN » : UN RITE DE PASSAGE OBLIGÉ}

« Nous, nous sommes opérationnels. On est sur le terrain, on connaît nos populations et on a une réelle force d'intervention. Les autres agences de l'Onu ne font

16 Un fonctionnaire international (40 ans), travaillant au HCR depuis 1998, rencontré à Genève en 2009.

17 Un fonctionnaire international (49 ans), travaillant au HCR depuis 1993, rencontré à Genève en 2008. 
que de l'appui institutionnel, elles restent dans les capitales et ont pour seuls interlocuteurs les États et non les populations cibles ${ }^{18}$.

L'identité professionnelle des fonctionnaires internationaux se construit autour d'un deuxième terme clé et tout aussi omniprésent dans les discours quotidiens : le « terrain ». À l'exception du Programme Alimentaire Mondial (PAM), le personnel perçoit en effet son institution comme la seule agence onusienne à être opérationnelle dans des zones souvent difficiles d'accès ou instables politiquement, ce qui renforce d'autant plus un certain sentiment de distinction vis-à-vis des autres agences de l'ONU. Au « terrain » est attaché un univers de sens fortement positif : il symbolise l'action, la flexibilité, l'esprit d'équipe, la solidarité, la proximité des populations bénéficiaires et la délivrance visible et concrète de services collectifs. Il révèle un univers de sens où l'efficacité des interventions se mesure avant tout par des réalisations visibles (telles que des infrastructures), quelles que soient leurs conséquences sur le long terme. Le « terrain » change de signification en fonction des situations de frontière. Si à l'échelle de l'institution, il permet de se distinguer face aux autres agences onusiennes, en son sein, il s'oppose au " siège », désigné par l'acronyme " $\mathrm{HQ}$ » (headquarters) qui n'est pas sans rappeler un certain vocable militaire. À l'échelle d'un pays donné, le «terrain » s'oppose au bureau de la " capitale », et se réfère dans ce cas aux antennes locales du HCR, implantées dans des zones reculées et parfois instables, au plus proche des camps de réfugiés. Aussi appelées non-family duty station, le staff n'est pas autorisé à s'y installer avec sa famille du fait de l'absence de services de base ou de conditions de sécurité jugées trop risquées. Il y vit, en général, en communauté au sein d'enceintes plus ou moins sécurisées où les conditions de vie sont sommaires, le stress très important et la séparation entre vie privée et vie professionnelle quasi-inexistante. Au siège comme aux capitales sont attribués des traits principalement négatifs : à l'instar des autres agences de l'ONU, ils symbolisent la « bureaucratie », l'« inertie », la « hiérarchie » ou encore des lieux de " paperasse » où l'on ne fait que rédiger des rapports d'activités et imposer de nouvelles procédures sans comprendre les réalités ni les contraintes du terrain. Ils incarnent aussi les lieux de pouvoir de l'organisation, là où se trouve la « direction », mais une direction perçue négativement parce qu'éloignée du terrain ${ }^{19}$.

Quelle que soit l'échelle où on se situe, le terrain renvoie finalement à l'idée que « c'est là où le vrai travail se fait » et à ce titre, il dénote une volonté affichée de se rattacher au monde de l'action humanitaire. Toutefois, tel n'a pas toujours été le cas, le travail juridique et de négociations diplomatiques ayant été, jusque dans les années quatre-vingt, bien plus valorisé que celui des opérationnels (Loescher, 2001). Le renversement des rapports de force à la faveur des humanitaires est donc désormais inscrit au cœur même des représentations du staff et l'on peut même avancer que le paradigme du « terrain » est devenu un mythe fondateur de l'identité professionnelle des fonctionnaires. Les modes d'interconnaissance s'opèrent à travers son prisme, et non pas celui de l'appartenance nationale ou culturelle : lors d'une première rencontre, on demandera ainsi à un collègue non pas de quelle nationalité il est, mais de quelle opération il vient. Le passage

18 Une fonctionnaire internationale (37 ans), travaillant au HCR depuis 2001, rencontrée sur le terrain en 2008.

19 Voir paragraphe sur le « senior staff management $»$. 
par le terrain a aussi acquis une dimension initiatique très forte, dont la reproduction est assurée par les aînés : "quand je lui ai confié mes craintes d'être envoyée au Darfour, mon superviseur m'a répondu que quand il était jeune, on l'avait envoyé en Bosnie au milieu de la guerre, alors il m'a dit que c'est comme ça si on veut travailler au HCR et que sinon on est «out» » ${ }^{20}$. Dans des non family duty station, la plupart des nouvelles recrues sont initiées aux discours, au travail et à l'esprit communautaire de l'institution : elles y apprennent ce que la protection signifie concrètement, rentrent en contact avec les réfugiés et se confrontent aux contraintes diplomatiques, logistiques et sécuritaires propres au mandat du HCR. Mais au-delà d'un certain savoir-faire, le déploiement sur le terrain permet aussi d'inculquer aux initiés un ensemble de manières de faire et de dire, ou encore de " savoir-être » et de " savoir-vivre » propres à l'institution (Cuche, 1988 :1) : cela inclut la capacité à être polyvalent et à gérer divers volets d'une opération dans un esprit de solidarité : "Sur le terrain, on est obligé de toucher à tout, car les équipes sont petites et chacun doit être solidaire, on fait de la protection, de l'administration, du programme... $»^{21}$; l'acquisition du principe de soumission à l'« urgence » et au travail intensif « sur le terrain, on travaillait 16 heures par jour toute la semaine, mais on sentait même pas la fatigue, c'était incroyable... $\gg^{22}$ et enfin la capacité à sacrifier sa vie personnelle pour partager avec ses collègues un mode de vie collectif et reclus. Le terrain apparaît donc à la fois comme un mode incontournable d'apprentissage du métier, mais aussi comme un rite de passage obligé pour être accepté au sein de sa communauté. On ne pourra être considéré comme membre à part entière de l'organisation et exercer par la suite une autorité considérée comme légitime sur ses pairs que si l'on a connu l'expérience des terrains difficiles. Plus qu'une séparation d'avec la famille ou le milieu social d'origine, ce rite permet donc d' " instituer une différence » (Bourdieu, $1986: 206$ ) entre ceux qui sont considérés comme les détenteurs d'une expertise légitime au sein de l'institution et les autres. Il classifie les individus suivant qu'ils aient acquis ce qui est perçu comme la «culture de l'organisation », ou qu'ils aient été parachutés par le haut, à des postes de direction sans connaître les « réalités du terrain». S'il participe à la production et la reproduction de l'autorité légitime au sein de l'organisation, ce rite signifie en même temps symboliquement et physiquement aux initiés ce qu'ils doivent être et ce qu'ils doivent faire pour faire partie du groupe. Il fonctionne donc également comme un « rite d'intensification » (Centlivres, 1986 : 193), en ce qu'il renforce le sentiment d'appartenance à un groupe social donné et l'intériorisation de ses valeurs dominantes (sens du sacrifice, polyvalence, solidarité, etc.) tout comme de ses mythes (ici, la conviction partagée que le HCR est une agence opérationnelle).

L'observation des pratiques montre néanmoins à quel point le « terrain » est une construction sociale qui permet de donner une image valorisante de son métier. Dans les non-family duty station en particulier, on constate en effet que ce n'est pas le personnel du HCR mais ses ONGs partenaires qui sont au plus près des réfugiés et vivent dans les

20 Une fonctionnaire internationale (34 ans), travaillant au HCR depuis 2003, rencontrée à Genève en 2009.

21 Une fonctionnaire internationale (37 ans), travaillant au HCR depuis 2001, rencontrée sur le terrain en 2008.

22 Un fonctionnaire international (40 ans), travaillant au HCR depuis 1998, rencontré à Genève en 2009. 
conditions les plus précaires et dangereuses. Ce sont elles qui sont chargées de la mise en œuvre concrète des programmes d'assistance au jour le jour, elles aussi qui enregistrent les réfugiés ou signalent les premières les cas de violations de leurs droits. En fait, le personnel du HCR tient plutôt un rôle de supervision et de suivi qu'il assure en se rendant dans les camps une à trois fois par semaine. De plus, les personnes chargées de ces missions sont généralement, soit les plus juniors parmi le personnel expatrié qui sont généralement sous contrats temporaires (« volontaires des Nations-unies » ou « jeunes professionnels »), soit le staff national recruté sur place. Les fonctionnaires internationaux ayant des postes stabilisés passent pour leur part une bonne partie de leur temps auprès des autorités locales à négocier la place du HCR à l'échelle locale, coordonner les activités avec les autres agences de l'ONU, résoudre des problèmes d'ordre logistique ou encore rédiger des rapports d'activités à destination de la capitale. Et plus ils avancent dans leur carrière, moins ils ont de contacts avec leurs bénéficiaires. Cette évolution est d'ailleurs souvent décrite avec regret et nostalgie par le personnel : « au Darfour, j'étais coincée dans des réunions inter-agences en permanence et dans l'écriture des rapports de réunions ; je n'avais plus le temps d'aller parler aux réfugiés et c'était très frustrant... ». Pourtant, au-delà de cette nostalgie affichée, on remarque qu'après avoir passé le stade de l'initiation et accepter plusieurs affectations difficiles, la plupart des fonctionnaires cherchent assez vite à rejoindre un bureau de capitale ou bien le siège, qui symbolisent pourtant tout ce qu'il y a de plus négatif et de dysfonctionnel au sein de l'organisation. On peut même avancer que le staff international se livre une concurrence très rude pour obtenir des places qui sont considérées comme chères, parce qu'en nombre plus limité que les postes en situation difficiles. Si elles ne sont pas dites explicitement parce qu'éloignées des valeurs dominantes de l'institution, les raisons en sont connues : avec le temps et l'évolution de sa carrière, le staff cherche d'un côté à se reposer ou à réconcilier vie privée et vie professionnelle, que ce soit pour mener une vie de famille normale ou pour tenter d'en construire une ; de l'autre, il espère également élargir ses réseaux au sein de l'institution, le passage par une capitale et à plus forte raison par le siège étant considéré comme indispensable pour " se faire connaître des directeurs, construire des réseaux et être soutenu tout au long de sa carrière $»^{23}$. Il existe ainsi un fort décalage entre ce qui est valorisé par le staff et placé au cœur de son identité professionnelle (le terrain), et les expériences vécues qui se marquent inévitablement par une distanciation croissante avec les populations bénéficiaires, une recherche de confort, voire même de sédentarité, et une volonté d'avancer dans sa carrière. Ce décalage doit se comprendre par la volonté de mettre en avant ce qui est perçu comme étant le plus « honorable » dans son travail par opposition au moins « respectable » (Hugues, 1996: 77) : tout discours sur son métier doit ainsi être analysé comme le véhicule d'un ensemble de jugements en termes de valeur et de prestige mais aussi comme une modalité de présentation d'une certaine image de soi. Dans la sphère humanitaire, auquel le staff du HCR s'identifie depuis la fin des années quatre-vingt, ce qui est le plus valorisé est donc le monde de l'action par opposition au monde de l'administration, et à l'instar de ce que Dauvin et Siméant ont pu dire des ONG, on observe aussi chez les fonctionnaires du HCR un même " complexe bureaucratique qui les oblige en permanence à célébrer le terrain » (Dauvin et Siméant, $2005: 356$ ), même si, au fur et à mesure de l'avancement de leur carrière, ils espèrent être affectés au siège.

23 Une fonctionnaire internationale (37 ans), travaillant au HCR depuis 2001, rencontrée sur le terrain en 2008. 


\title{
« LA MAISON » : LE PARTAGE D'UNE VIE COMMUNAUTAIRE
}

\author{
"Nous formons ici une grande famille et la plus grande force de notre maison, \\ c'est son personnel. Ce sont des jeunes et des moins jeunes flexibles, compétents, \\ dynamiques, dévoués, prêts à partir et s'engager sur le terrain, et surtout prêts à \\ accepter beaucoup de frustrations et de sacrifices $\|^{24}$.
}

Un troisième terme récurrent dans le langage quotidien du staff international est celui de "maison » ou "house » auquel se substitue dans certains cas la notion encore plus forte de « famille ». Avec le « mandat» et le « terrain », la « maison » est souvent identifiée comme la troisième grande force de l'institution. Elle est utilisée de manière métaphorique en référence au logo du HCR symbolisant une personne protégée par un toit. Toutefois, si le logo désigne un réfugié, le personnel utilise le terme de «maison» pour s'auto-désigner. La maison ne protège donc pas, dans cette acceptation, les réfugiés, mais le staff. Elle symbolise à elle seule le sentiment d'appartenir à une même communauté à laquelle est attribué un ensemble de qualités fortement valorisées par le groupe, telles que la compétence, la polyvalence et surtout le sens du sacrifice et du dévouement. Ce sentiment se construit à la fois sur la volonté de se distinguer par rapport aux Autres, et sur l'expérience concrète de la vie communautaire au sein des affections difficiles et du partage de repères spatio-temporels similaires.

Les fonctionnaires du HCR se rejoignent en effet autour d'un même mode de vie, construit à cheval entre le milieu des expatriés intervenant plus largement dans le champ du développement ou de la coopération, et le milieu des humanitaires et des volontaires, déployés sur de courtes périodes dans des zones risquées ou difficiles d'accès. Capables de mener des négociations de haut niveau avec des représentants gouvernementaux au Palais des Nations à Genève et de se fondre dans les milieux diplomatiques, ils savent aussi coordonner des programmes d'urgence dans le Kivu avec les moyens du bord et s'accommoder de conditions de vie sommaires. Ils oscillent donc sans cesse entre des univers sociaux et culturels très différents, au sein desquels ils mobilisent des compétences, des codes de conduite et des codes vestimentaires spécifiques. Se qualifiant eux-mêmes de mélange entre «des cow-boys, des nones et des diplomates », ils se situent ainsi dans un espace d'entre d'eux, qui exprime le tiraillement chronique que connaît le HCR entre son identité d'agence des Nations Unies et sa vocation (récente) d'agence humanitaire. Si le HCR n'est pas le seul à occuper cet espace d'entre d'eux sur la scène internationale ${ }^{25}$, le staff international en retire néanmoins un sentiment subjectif très fort de " distinction » qui lui permet de construire son identité propre dans le champ institutionnel fortement concurrentiel qu'est celui de l'humanitaire. D'un côté, nous avons déjà vu que les fonctionnaires cherchent à se différencier des autres agences de l'ONU et du monde de la bureaucratie, en se rattachant au monde de l'action et du terrain - «nous sommes plus opérationnels que les autres agences de l'ONU, nous au moins nous faisons du concret

24 Un fonctionnaire international (44 ans), travaillant au HCR depuis 1997, rencontré à Genève en 2007.

25 On peut par exemple penser au PAM, mais aussi à l'UNICEF et plus récemment OCHA. 
et pas seulement de la paperasse ou de beaux discours $»^{26}$. Mais de l'autre, ils cherchent en même temps à se distinguer des ONG humanitaires, en revendiquant la spécificité de leur autorité diplomatique et de leur professionnalisme, par opposition au militantisme associatif et à la faiblesse des moyens des acteurs non-gouvernementaux : " contrairement aux ONG, nous avons plus de poids face aux autorités pour imposer certaines choses et nous ne nous limitons pas à critiquer ou dénoncer des problèmes complexes sans même les maîtriser. Nous faisons de la real politik $»^{27}$. Outre la dimension morale de leur autorité, déjà évoquée, le sentiment d'avoir plus de poids par rapport aux ONG se matérialise aussi à travers un ensemble de signes distinctifs tels que le statut de fonctionnaire international, incarné matériellement par le fait de détenir un "UN laissez passer » - équivalent à un passeport diplomatique attribué au staff international des Nations Unies ; d'être véhiculés dans des énormes véhicules $4 \times 4$ Toyota Land cruiser, réputées comme les plus solides mais aussi les plus chères; ou encore de travailler dans l'enceinte des larges bâtiments des Nations Unies, reconnaissables par leur couleur bleue et blanche, leur barbelés, leur garde et leur drapeau, etc. Si ces insignes font souvent l'objet de critiques virulentes de la part des ONG (Dauvin et Siméant, 2005 : 303), ils procurent à leurs détenteurs le sentiment d'appartenir à une élite dotée d'une légitimité universellement reconnue et ils consacrent ainsi la frontière entre eux et les organisations non-gouvernementales. Mais cet élitisme s'exprime aussi par rapport à tous les « Autres » au sens large, c'est-à-dire à ceux qui n'appartiennent pas au monde de l'expatriation, de la diplomatie ou de l'humanitaire, et dont les modes de vie sont perçus comme routiniers, ternes et sans enjeux. Il existe en effet une perception partagée - souvent entretenue par des effets de miroir par l'entourage familial ou amical - de vivre des situations qui sortent de l'ordinaire, que ce soit lorsque l'on se trouve au cœur du Darfour ou du Kivu dans les avions spéciaux des Nations Unies, ou au cœur des hémicycles du Palais des Nations à Genève entouré de chefs de missions diplomatiques du monde entier. Le sentiment élitiste se construit ainsi avant tout «par défaut, en méprisant les façons de faire des voisins » (Dauvin et Siméant, $2005: 194$ ) et ceci à travers un triple rejet : celui de la lourdeur de la bureaucratie onusienne, de la légèreté du militantisme associatif des ONG et de la banalité d'une vie sédentarisée et sans aventure. Toutefois, il se construit aussi en ne sélectionnant que les aspects positifs de la double appartenance au monde onusien (le pouvoir et la légitimité) et au monde de l'action (l'efficacité et la proximité avec les populations bénéficiaires).

La perception d'appartenir à une même communauté se construit aussi à travers l'expérience de vie collective dans les affectations difficiles. Là, nous avons vu que les fonctionnaires partagent une même maison, au sens premier du terme, pour des périodes allant jusqu'à deux ou trois ans. Les « compounds» et les « guest houses $»^{28}$ deviennent alors de véritables foyers de substitution l'espace de quelques temps : « Sur le terrain, il

26 Une fonctionnaire internationale (37 ans), travaillant au HCR depuis 2001, rencontrée sur le terrain en 2008.

27 Une fonctionnaire internationale (34 ans), travaillant pour le HCR depuis 2003, revenant du terrain et rencontrée à Genève en 2009.

28 Suivant la phase de sécurité établie par l'ONU, le staff peut soit vivre et travailler dans une même enceinte (appelée « compound») ou bien avoir un lieu de vie communautaire séparé du lieu de travail (appelé dans ce cas «guest house »). Lorsque les conditions de sécurité le permettent, le personnel peut aussi être autorisé à louer sa propre maison dans son lieu d'affectation.

REMI 2009 (25) 3 pp. 167-190 
n'y a plus de séparation entre vie privée et vie professionnelle; on mange, on travaille, on dort ensemble; on est jamais seul. Les conditions de vie et de sécurité sont souvent très dures mais on est entouré de gens dynamiques, enthousiastes et plein de vitalité, et on crée souvent des liens très forts "). Les espaces privés se limitent à une chambre dans l'enceinte collective et la vie quotidienne à un travail intensif dans des conditions souvent très contraignantes. Les déplacements sont soumis à un contrôle très strict de la part de la sécurité des Nations Unies et les loisirs s'arrêtent à des soirées, souvent bien arrosées et très festives, au sein même du compound. Le ravitaillement est généralement assuré par le personnel recruté sur place et le staff complètement pris en charge. Les fonctionnaires internationaux connaissent ainsi une forme d' " enfermement symbolique » (Bourdieu, 1988 : 121) ${ }^{29}$ où l'emprise de l'institution et des liens communautaires sur l'individu atteint son paroxysme. Décrivant les missions humanitaires des ONG, Dauvin et Siméant va jusqu'à comparer ce type d'expériences communautaires à celles des institutions totales telles que l'armée, les couvents ou le scoutisme (Dauvin et Siméant, 2005 : 293). Outre sa dimension initiatique et son rôle de transmission des valeurs de l'institution, cet enfermement génère la construction de relations affectives intenses avec ses pairs, et contribue à façonner un certain esprit de solidarité et de fraternité face à des conditions perçues comme de l'ordre de l'adversité. La vie communautaire rend aussi le retour vers les capitales souvent difficile, même s'il est, paradoxalement, activement recherché. "Je voulais aller à Genève pour me reposer, mais l'arrivée ici a été très dure, j'avais tout le temps envie de pleurer. Le terrain procure une forme d'adrénaline. On prend l'habitude de vivre sous une pression permanente et en communauté. Et on n'est jamais seul, et on est pris en charge. Beaucoup de collègues ont peur de devoir revenir à une vie normale et c'est comme ça qu'ils vont de crises humanitaires en crises humanitaires $»^{30}$. Quel que soit le lieu de l'affectation, on remarque finalement une certaine insatisfaction chez le personnel du HCR : sur le terrain se fait sentir le besoin de renouer avec une vie plus calme et facile, mais dès que celle-ci est retrouvée, la nostalgie pour les sensations fortes et la vie communautaire se fait sentir, de même que la difficulté à renouer avec une forme de sédentarité. Partager ses ressentis avec des personnes n'ayant pas connu les mêmes expériences devient aussi de plus en plus difficile. Le HCR devient alors progressivement l'espace principal de socialisation et de construction identitaire : aux réseaux sociaux construits avant l'entrée au sein de l'organisation se superposent - voir se substituent progressivement des liens très forts avec ses collègues : " moi, aujourd'hui, j'ai plus de choses à partager avec des gens qui ont une culture de l'expatriation et de l'humanitaire qu'avec ceux de ma propre nationalité ou qu'avec ma propre famille $»^{31}$. Cette situation se traduit par une endogamie relativement importante au sein même du HCR ou du cercle plus large des humanitaires, mais aussi par un taux de célibat ou de divorce relativement élevé (Wigley, 2005). Elle explique aussi pourquoi le sens du sacrifice du personnel est si fortement valorisé par les fonctionnaires, et crée, en retour, des attentes importantes envers

29 Bourdieu utilise ce terme dans La Noblesse d'État (1988), en référence au système français des " classes préparatoires », qui offrent un enseignement intensif aux étudiants souhaitant présenter les concours d'entrée aux écoles françaises les plus prestigieuses.

30 Une fonctionnaire internationale (34 ans), travaillant pour le HCR depuis 2003, revenant du terrain et rencontrée à Genève en 2009.

31 Une fonctionnaire internationale (37 ans), travaillant pour le HCR depuis 2001, rencontrée sur le terrain en 2008. 
l'institution, notamment en termes de reconnaissance et de promotion.

Le sentiment d'appartenir à une même maison se construit enfin sur le partage d'un même rapport au temps et à l'espace, caractérisé par une tension constante entre une dimension déterritorialisée, liée au partage d'un même calendrier de travail et de festivités, et une dimension hyper-territorialisée, liée aux changements d'affectations géographiques et aux urgences humanitaires. Le découpage de l'espace se calque tout d'abord sur les oppositions entre le siège, les capitales et le terrain, ainsi que sur un certain nombre de zones " chaudes ", où se déploient les opérations du HCR les plus importantes et les plus médiatisées du temps présent. L'espace se dessine ainsi sur une toile d'îlots de prospérité et de crises humanitaires, dont les emplacements changent en fonction de l'actualité politique et de l'ouverture ou de la fermeture des antennes du HCR de par le monde. Il s'agit d'une espace mouvant et éphémère, où le seul point de repère stable est Genève, perçu avant tout comme un « siège » international, et non comme un lieu géographiquement ancré sur le territoire suisse. Dans cet espace, le rapport au temps se calque sur la toute puissance du temps présent, de l'événement et de l'urgence. À l'échelle individuelle, il est d'abord déterminé par le cycle des affectations géographiques qui oscille de 2 à 5 ans : celles-ci deviennent des repères fictionnels essentiels dans la construction des récits biographiques même si parfois l'ordre de leur succession se brouille dans les mémoires. Chaque nouvelle prise de fonction est vécue sur le mode de la découverte, d'un nouveau contexte professionnel mais aussi d'un nouveau pays : "ce que je préfère au HCR et ce pourquoi je reste malgré certaines frustrations, c'est que l'on ne s'ennuie jamais, chaque fois, c'est une nouvelle vie qui démarre $»^{32}$. À l'échelle collective, le temps se construit au contraire dans un cadre déterritorialisé : il est ponctué par une succession d'événements, qui mobilisent l'ensemble du personnel, quelle que soit sa fonction ou son affectation. Bien qu'ils se renouvellent annuellement, ces événements sont toujours traités sur le mode de l'urgence, qu'il s'agisse de la préparation, en mars du document stratégique de planification des opérations pour l'année suivante (le " country operation plan »), de celle, en juin, de la Journée Mondiale du Réfugié, pour laquelle chaque antenne doit organiser des festivités, ou encore de l'organisation, en octobre, de l' «EXCOM », acronyme faisant référence au comité exécutif du HCR rassemblant chaque année les 72 États-membres de l'organisation suivant une procédure extrêmement protocolaire (Fresia, à paraître). Bien d'autres événements, liés au cycle des opérations et à la célébration des «Journées mondiales » des droits de l'homme ponctuent la vie des fonctionnaires internationaux. À cela s'ajoutent des moments très ritualisés liés à la célébration des héros et des martyrs de l'institution. La fin de l'année se clôture ainsi par la remise du prix « Nansen »- du nom du tout premier Haut commissaire pour les réfugiés qui reçut le prix Nobel de la paix en 1927 - décerné à une personnalité s'étant démarquée pour son engagement pour la cause des réfugiés. Mais à d'autres moments, on se recueille de par le monde à l'annonce d'une prise d'otage ou du décès d'un collègue sur le terrain, mort pour défendre cette même cause $^{33}$. Ces événements sont autant d'occasions de se réunir pour célébrer la « maison» et ses valeurs et renforcer le sentiment de distinction vis-à-vis de l'extérieur. Au sein de

32 Une fonctionnaire internationale (34 ans), travaillant pour le HCR depuis 2003, revenant du terrain et rencontrée à Genève en 2009.

33 Un mémorial en l'honneur des martyrs du HCR a même été dressé, au début des années deux mille, devant le siège de l'organisation à Genève.

REMI 2009 (25) 3 pp. 167-190 
la « maison », on notera enfin, sans développer, que le staff évolue au sein d'un même univers sémantique et partage un même langage ésotérique qui incorpore une variété de concepts, de termes techniques et d'acronymes - désignant les standards, outils, procédures et départements de l'institution - sans cesse réinventés en fonction des nouvelles orientations politiques de l'organisation. Si la mobilisation quotidienne de ce jargon revêt une dimension stratégique orientée vers la mobilisation de fonds, elle tend parfois aussi à devenir le seul moyen de communiquer avec ses collègues mais aussi la seule grille de lecture utilisée pour interpréter la complexité du réel. Support d'un même cadre cognitif, le langage de la « maison » contribue ainsi à influencer la perception du monde tout comme les rapports sociaux, et participe aussi pleinement de la construction d'un sentiment d'appartenance collective (Kunda, 1992).

Au-delà de sa représentation idéalisée comme lieu de solidarité, de réconfort ou de valeurs partagées, la " maison » reste néanmoins traversée quotidiennement par de multiples conflits qui animent d'ailleurs nombre d'intrigues et de discussions. Au niveau vertical, des rapports de pouvoir, de compétition et d'envie apparaissent ainsi à chaque niveau de la hiérarchie organisationnelle (Wigley, 2005). Malgré plusieurs tentatives de réformes, le HCR reste une organisation de type pyramidale, fondée sur des figures du pouvoir très personnalisées, avec à sa tête celle du "Haut Commissaire »-aussi appelé «HC », qui est elle-même incarnée à l'échelle de chaque pays par celle du " Représentant» ou « Rep ». Sous l'autorité de ce dernier opère le personnel international (professionnal staff ou "P staff ») qui est lui-même supérieur au personnel national (general staff ou "G staff »). Chaque catégorie est également sub-divisée en 5 ou 6 grades (P2, P3, etc.), auxquels correspondent des niveaux de rémunération, des tâches mais aussi des conditions de travail différents ${ }^{34}$. Ces distinctions catégorielles sont source de fortes stigmatisations et génèrent, surtout, une concurrence importante entre les fonctionnaires pour qui l'avancement dans la hiérarchie est conçu avant tout comme une valorisation des sacrifices consentis pour l'institution. Au niveau horizontal, les conflits sont tout autant, sinon plus nombreux : la « maison » est également saucissonnée et sectorisée, en particulier depuis son engagement dans les activités humanitaires de grande envergure depuis la fin des années quatre-vingt. Trois grands piliers la divisent à chaque niveau institutionnel depuis le siège jusqu'au terrain : le département de la « Protection », celui des « Opérations », et celui de l' "Administration », reflets de la coexistence entre plusieurs cultures professionnelles au sein de l'organisation. Les Opérations se découpent elles-mêmes en zones géographiques distinctes et sont supervisées à Genève par les «Bureaux » pour l'Asie, l'Afrique, l'Europe et les Amériques. Or départements et zones géographiques définissent chacun leurs objectifs en ignorant ceux des autres, et entrent aussi en compétition à la fois autour de la définition et de l'interprétation des politiques de l'organisation, mais aussi de la répartition interne de ses ressources. Chacun s'engage ainsi dans de véritables luttes symboliques pour gagner en visibilité et déploient pour cela une variété de stratégies qui prennent souvent beaucoup de temps et d'énergie, au détriment de l'objectif principal de l'organisation. Elles sont particulièrement intenses à Genève, où le saucissonnage des activités a atteint son plus haut niveau depuis les années quatre-vingt-dix avec la multiplication des sections techniques chargées de produire des standards pour chaque secteur de l'assistance et chaque catégorie de victimes. Sans surprise, on constate alors que, comme

34 À Genève, un P3 aura par exemple un bureau plus grand et une chaise plus confortable qu'un P2. 
pour toute bureaucratie, les fonctionnaires tendent à s'enfermer dans la défense de leur seul sous-objectif et secteur d'intervention, travaillant ainsi les uns contre les autres et non pas les uns avec les autres au service d'un objectif commun plus large : « de l'idée d'objectifs fixés suite à une décision rationnelle de la direction (...), on passe alors à celle d'objectifs définis dans la confrontation d'acteurs en quête d'autonomie ou d'influence » (Bagla, 2003 : 75). Les politiques, les normes mais aussi les valeurs défendues par l'institution et présentées comme universelles et consensuelles sont donc toujours, en réalité, le résultat de configurations de pouvoir complexes : elles ne reflètent, en pratique, que les intérêts et les représentations d'un nombre limité d'acteurs en son sein.

\section{«LE SIÈGE » : L'EXÉCUTOIRE DE FRUSTRATIONS PARTAGÉES}

Si elle se fonde sur un ensemble de référents positifs (le mandat, le terrain et la maison) qui expriment un sentiment de distinction vis-à-vis des autres et une vision idéalisée de leur institution, l'identité professionnelle des fonctionnaires du HCR se construit également sur un ensemble de frustrations partagées, que le siège à lui tout seul semble concentrer. Ces frustrations se déclinent autour de plusieurs expressions dont on ne sélectionnera ici que les plus significatives.

\section{- DHRM}

«Le problème principal du HCR, c'est DHRM. Le système des promotions et des affectations est complètement inéquitable, dysfonctionnel, opaque et clientéliste.

Certains sont toujours affectés dans des pays difficiles et d'autres restent dans les capitales ou à Genève ; et certains connaissent une ascension fulgurante tandis que d'autres stagnent pour un niveau de compétence équivalent $»^{35}$.

« DHRM » (Division for Human Ressource Management) est l'acronyme désignant le département des ressources humaines du HCR. Il apparaît comme la bête noire du staff pour qui la gestion des rotations et des promotions est à la fois la principale faiblesse de l'institution et source de désillusions à l'échelle personnelle. En principe, le système est basé sur l'équité (alternance entre affectations difficiles et moins difficiles), la compétence (adéquation des profils et des fonctions), le mérite (évaluation des performances) et la transparence (décisions prises par une commission mixte représentant le conseil du personnel, la direction et les ressources humaines). Le staff n'est donc pas libre de choisir où il sera envoyé même s'il établit une liste de postes désirés par ordre de priorité. Les normes officielles sont toutefois court-circuitées par trois éléments au moins : (i) par d'autres règles officielles formulées par d'autres sections du HCR telle que la promotion genre par exemple ou l'obligation de placer en priorité le staff sans affectation depuis plusieurs mois ; (ii) par des normes officieuses telles que le clientélisme, les réseaux d'interconnaissance, le pouvoir discrétionnaire de certains directeurs, la nationalité ${ }^{36}$, ou l'absence de crédibilité accordée aux modalités d'évaluation des compé-

35 Un fonctionnaire international (49 ans), travaillant au HCR depuis 1993, rencontré à Genève en 2008.

36 Les gros bailleurs de fonds faisant pression pour favoriser la carrière de leurs ressortissants. 
tences ; (iii) par des normes d'équité implicites qui veulent que les plus jeunes «payent pour la retraite » des plus vieux en étant affectés prioritairement dans des non-family duty station. Il y a donc une multiplicité de normes qui se court-circuitent les unes les autres et qui, de plus, ne cessent d'évoluer au cours de temps. Ce pluralisme normatif confère une certaine marge de manœuvre à DHRM dans l'interprétation des règles et engendre, dans certains cas, une inadéquation entre les compétences des individus et les fonctions sur lesquelles ils sont nommés. Ainsi, on constate paradoxalement que ce sont souvent les plus jeunes - donc les moins expérimentés - mais aussi les managers les moins compétents (dont personne ne veut) qui sont affectés dans les opérations de terrain les plus difficiles, les moins médiatisées ou les moins soutenues par le siège. De même, pour un niveau égal de compétences, certains obtiennent des promotions très rapidement - parce qu'ils ont la bonne nationalité, le bon réseau ou le bon genre - tandis que d'autres restent au même grade pendant des années. Cette situation entretient chez le staff un fort sentiment d'opacité et d'arbitraire dans les choix opérés par les ressources humaines, et la conviction largement partagée que celles-ci mettent en avant des critères peu légitimes (les réseaux de clientèles, le genre, l'âge ou l'origine) par rapport à ce que le staff considère être les vrais valeurs de l'organisation (le sens du sacrifice, la compétence, la polyvalence). La politique des ressources humaines anime nombre de conversations quotidiennes au sein du HCR et contribue aussi, d'une certaine manière, au sentiment de partager un même destin, placé cette fois-ci sous le signe de l'injustice et non pas de la distinction. Elle suscite, surtout, fatigue, colère et frustration parmi ceux toujours affectés dans des opérations difficiles ou qui n'obtiennent pas les promotions estimées comme « justes » aux yeux des sacrifices consentis pour l'organisation. Le staff perçoit en effet l'avancement dans la hiérarchie et la possibilité d'obtenir, à un moment donné de sa carrière, le poste de son choix comme une forme de contrepartie que l'institution lui doit en échange de la soumission au principe d'affectation dans des non-family duty station et aux difficultés endurées sur le terrain. Non satisfait, ce besoin de reconnaissance génère de forts ressentiments non seulement à l'égard de l'institution, mais aussi des réfugiés. Plusieurs recherches sur le travail humanitaire ont ainsi montré comment le personnel humanitaire, par un processus de transfert des responsabilités, tend à rendre les réfugiés responsables de leurs propres désillusions qui sont liées, en réalité, aux dysfonctionnements du système de l'aide (Walkup, 1997 ; Harrell-Bond, 1986).

\section{- Le senior management committee}

"On a l'impression de se faire diriger par une clique qui est de plus en plus déconnectée du terrain et qui n'a pas la culture de l'organisation $»^{37}$.

Le « senior management committee » est une autre source de mécontentement parmi le personnel, qui révèle également la relation que les fonctionnaires entretiennent avec le pouvoir. Cette expression désigne au sens restreint le Haut Commissaire, entouré de trois hautes personnalités chargées de superviser les trois grands piliers du HCR : l'Assistant au Haut commissaire pour les opérations ; l'Assistant au Haut Commissaire pour la Protection, et l'Adjoint au Haut commissaire pour l'administration. Au sens plus large,

37 Une fonctionnaire internationale (37 ans), travaillant au HCR depuis 2001, rencontrée sur le terrain en 2007. 
elle se réfère à l'ensemble des personnes occupant des postes de direction et comprend aussi les directeurs des bureaux régionaux et les représentants du HCR dans chaque pays. Les directeurs souffrent d'une très mauvaise réputation au sein de l'organisation, en particulier ceux qui ont été parachutés par le haut et nommés pour des raisons politiques. De nombreuses personnes évoquent ainsi "une tête coupée du corps», et selon certaines, cette tendance irait en s'accentuant : " il y a eu une perte de direction dernièrement; avant ceux qui prenaient des décisions connaissaient les réalités opérationnelles du HCR et le terrain. Mais aujourd'hui, les décideurs sont des gens qui ne connaissent rien des opérations et du système de planification propre à l'organisation et ils sont en train de tout détruire $»^{38}$. Le senior management committee ne dispose donc pas d'une autorité considérée comme légitime : il n'est pas intégré comme membre à part entière de la « maison », parce qu'il n'a pas intégré ce que le staff considère être la «culture de l'organisation » ni connu les mêmes rites de passage. Ce manque de légitimité se reflète à travers une série de reproches formulée à son encontre : il n'agirait que pour ses intérêts propres en se soumettant aux demandes des bailleurs de fonds et il serait, de plus, divisé, les directeurs de chaque grand pilier travaillant les uns contre les autres et non pour l'intérêt général de la maison. Plus significatif encore, depuis la prise de fonction du Haut Commissaire Gutteres en 2005, les dirigeants sont désormais qualifiés de simples « technocrates ». D'une part, on les accuse d'alourdir toujours plus les contraintes qui pèsent sur le terrain et la bureaucratisation de certaines activités, au nom des principes de " rationalisation » et de « transparence » imposés depuis le début des années deux mille par les bailleurs de fonds. De fait, la direction exige désormais du staff qu'il entreprenne une « gestion basée sur les résultats » (result-based management) : celle-ci se traduit concrètement par l'imposition de nouveaux indicateurs quantitatifs, cadres logiques et logiciels devant permettre de mesurer les progrès effectués pour atteindre des objectifs prédéterminés - une tendance que l'on retrouve dans toutes les administrations publiques aujourd'hui et qui est porteuse d'une certaine idéologie politique (inspirée du néo-libéralisme et de la planification technocratique) et de certains présupposés positivistes (Giovalucchi et Olivier de Sardan, à paraître). D'autre part, la direction, qui a aussi entamé un processus de délocalisation et de décentralisation de certaines fonctions pour diminuer les coûts administratifs de l'institution, est aussi accusée de vouloir " dépolitiser » et « désyndicaliser » le staff en le mettant dans l'insécurité. Si le HCR est en réalité en réforme permanente - trois restructurations majeures ont déjà été entreprises depuis la fin des années quatre-vingt (Loescher, 2001), le staff la perçoit comme inédite : elle marquerait en effet une nouvelle reconfiguration des rapports de force au sein du HCR, en faveur cette fois-ci de la sphère administrative et managériale et donc du troisième pilier de l'institution. Cette perception cristallise aussi les déceptions : "Quand je suis rentré aux Nations Unies, je pensais qu'on y défendait des valeurs sociales progressistes ; mais je constate que derrière leurs beaux slogans, nos dirigeants sont les premiers à se soumettre aux impératifs du néo-libéralisme ${ }^{39}$.

Au sein de l'institution, les fonctionnaires internationaux construisent donc aussi leur identité sur le rejet de la direction, qui leur permet de transcender leurs divisions

38 Un fonctionnaire international (40 ans), travaillant au HCR depuis 1998, rencontré à Genève en 2009.

39 Un fonctionnaire international (38 ans), travaillant au HCR depuis 1999, rencontré à Genève en 2009. 
internes. La direction et plus largement le siège semblent même servir d' « exécutoire » aux frustrations générées par l'exercice quotidien de sa profession. Ils incarnent tout ce qu'il y a de plus dévalorisant pour l'institution : la bureaucratisation, l'inefficacité, le clientélisme ou encore la tendance à se soumettre aux exigences des bailleurs de fonds ou aux préceptes du néo-libéralisme. Pourtant, la frontière entre la direction et le staff - tout comme le siège et le terrain - est en réalité loin d'être aussi nette qu'il n'y paraît. Au-delà des représentations que les acteurs s'en font, leurs relations doivent être envisagées dans un rapport dialectique et non pas d'opposition. D'une part, il faut rappeler que ce sont les mêmes personnes qui circulent entre le siège et le terrain. Or, on constate que lorsque les fonctionnaires sont affectés au siège et nommés à des postes administratifs ou techniques, ils sont les premiers à participer à la bureaucratisation et la rationalisation des activités du $\mathrm{HCR}$, et à promouvoir le principe du « result-based management » en contribuant - au sein de leur unité - à produire des documents stratégiques, des objectifs, des standards et des indicateurs à respecter pour chaque domaine d'intervention. D'autre part, on observe que les lieux de pouvoir au sein du HCR sont beaucoup plus segmentés et diffus que la structure officielle ne le laisse croire. Si les grandes orientations politiques et managériales impulsées par la direction peuvent avoir une grande influence sur l'ensemble de l'organisation, elles font aussi l'objet de constants processus de réappropriation et de fragmentation à diverses échelles institutionnelles, limitant ainsi leur portée réelle ou la modifiant. Si l'on reprend l'exemple des procédures et des standards imposés par le siège, on remarque en effet que sur le terrain, nombre d'entre eux ne sont parfois tout simplement pas connus du personnel opérant sur le terrain, parce que pas toujours relayés par les « capitales ». Et lorsque certaines procédures sont connues de tous de par leur caractère obligatoire, on constate que le staff international s'y soumet à la va-vite : les rapports d'activités sont par exemple rédigés sous forme de " copier-coller » et ont moins une fonction de rationalisation de l'activité de planification, que de légitimation des budgets demandés et de visibilité auprès du siège. Autrement dit, de nombreuses décisions prises par la direction sont soit court-circuitées soit réinterprétées par différents maillons de la chaîne, qui en font un autre usage de ce à quoi elles étaient destinées. Comprendre la relation dialectique entre le siège et le terrain, la direction et le staff suppose donc aussi de prendre en compte le rôle des intermédiaires qui se situent aux interfaces entre différents niveaux institutionnels et disposent d'un certain pouvoir d'influence pour orienter les impulsions politiques données par le « haut ». Parmi eux, on peut citer par exemple les « Représentants » ou les chefs de bureaux qui relaient les décisions de Genève ou de la capitale à l'échelle locale, mais aussi les " sédentaires », c'est-à-dire le staff national, qui maîtrisent les rouages de chaque opération et font office de mémoire institutionnelle, ou encore le "junior staff» et les ONG partenaires du HCR qui sont en contact direct avec les réfugiés sur le terrain. La compréhension de la relation siège-terrain suppose aussi de s'intéresser au rôle des réseaux interpersonnels ou de clientèle qui la traversent : les réseaux sont effectivement indispensables pour gérer sa carrière mais aussi pour faire son travail au sein d'une organisation aussi vaste et mouvante que le HCR. Certains se construisent de manière spontanée au sein d'une même opération (réseaux d'anciens combattants), d'une même classe d'âge (réseau des jeunes professionnels) ou d'une même langue (réseau francophone), et d'autres de manière activement recherchée via le déploiement de diverses stratégies (partir en mission au siège où sont concentrés de nombreux directeurs ; suivi de formations internes pour rencontrer d'autres collègues, etc.). Ainsi, bien qu'il dénonce les pratiques clientélistes du siège et en particulier de DHRM, le staff est contraint d'y participer lui-même pleinement, 
ne serait-ce que pour négocier sa prochaine affectation ou promotion (« si on connaît personne aux ressources humaines ou à la direction, on a aucune chance, si ce n'est d'être envoyé au fin fond $d u$ Tchad $»^{40}$ ) mais aussi pour contourner quotidiennement un ensemble de contraintes internes à l'institution et pour " personnaliser» des relations de travail qui resteraient autrement impossibles à nouer. Comme pour d'autres institutions, on devine ainsi l'existence d'un écart important entre lieux de pouvoir formels et informels, les règles, les fonctions, et les compétences étant sans cesse négociées et construites au sein d'interactions quotidiennes entre des acteurs en situation (Strauss et al., 1963).

\section{CONCLUSION}

En prenant pour porte d'entrée l'analyse de certains termes clés mobilisés au quotidien par les fonctionnaires du HCR, cet article illustre la manière dont une élite transnationale se fabrique un univers de sens partagé et une identité professionnelle qui permettent de créer du " lien » par-delà son hétérogénéité culturelle et sa dispersion géographique. Cette identité professionnelle s'articule autour de trois dimensions au moins : (i) une dimension morale et idéologique, liée au partage d'un cadre cognitif commun et à la conviction d'agir pour le bien de l'humanité ; (ii) une dimension communautaire, qui se manifeste par le partage d'expériences collectives parfois très ritualisées, au cours desquelles les membres de cette élite développent des liens de solidarité et s'approprient un ensemble de valeurs, de manières de dire et de faire similaires ; (iii) et une dimension élitiste, qui se construit sur l'appropriation d'un double statut : celui du « fonctionnaire international » qui confère à sa profession une légitimité perçue comme universelle, et donne accès aux plus hautes sphères du pouvoir à l'échelle mondiale; et celui $\mathrm{du}$ « travailleur humanitaire », qui permet de se rattacher au monde de l'action et de l'efficacité par opposition à celui de la bureaucratie et de l'inertie onusiennes. Le sentiment d'appartenance collective se forge ainsi sur un mélange d'élitisme, de communautarisme et de moralisme, et sur différentes « étiquettes professionnelles » qui sont mobilisées différemment en fonction des situations de frontière (suivant que l'on cherche à se distinguer des autres agences de l'ONU, des ONGs ou des Autres au sens large). Loin d'être une donnée en soi, il se construit au jour le jour à travers des interactions et des réseaux d'interconnaissance au sein desquels sont partagés des événements émotionnellement très forts sur le terrain, mais aussi un ensemble de dilemmes liés à la difficulté de réconcilier vie privée et vie professionnelle, goût pour une certaine forme d'aventure et recherche de stabilité, défense des droits des réfugiés et défense du droit à la vie. Les frustrations générées par ces dilemmes se cristallisent le plus souvent contre l'institution, symbolisées par la figure du « siège » et du « senior management committee » qui font office d'exécutoire, mais parfois aussi contre les réfugiés eux-mêmes. Elles sont sous-tendues par une tension constante entre une représentation idéalisée de l'organisation et de ses valeurs, et des expériences vécues dans l'exercice quotidien de sa fonction, qui génèrent déception et désillusions. Ainsi la fabrique d'une même communauté d'appartenance n'amène pas le personnel à s'identifier à l'institution de manière uniquement positive : celui-ci reste au contraire très critique et réflexif vis-à-vis d'elle. Toutefois, ces critiques se focalisent plus

40 Une fonctionnaire internationale (36 ans), travaillant au HCR depuis 2004, rencontrée à Genève en 2009. 
sur les dérives internes à l'institution - lorsqu'elles vont à l'encontre de ce qui est valorisé par le staff - que sur la portée externe de ses interventions, pourtant fortement contestée par de nombreuses ONG mais aussi par le milieu scientifique. Si certains reconnaissent que le HCR ne s'oppose pas assez fermement aux États peu respectueux de leurs obligations internationales, la plupart des fonctionnaires restent en effet convaincus que « mieux vaut changer les choses de l'intérieur » et faire de la " real politik» que de rester dans une position d'externalité. Le réalisme l'emporte donc le plus souvent sur le militantisme politique.

La fabrique d'une identité professionnelle permet finalement d'institutionnaliser une relation subjective entre les membres du HCR et de produire un certain « ordre » institutionnel, même si celui-ci n'est pas antinomique avec l'existence de fortes tensions et de négociations quotidiennes du sens donné à son travail, à sa fonction et plus largement à la mission de l'organisation. L'analyse de ce processus reste néanmoins encore largement à approfondir : des études de cas, à diverses échelles d'observation, nous permettraient par exemple de mieux comprendre comment cette identité collective - et les valeurs qu'elle véhicule - s'actualise dans des configurations ou autour d'enjeux spécifiques, de même qu'une étude fine des trajectoires personnelles des fonctionnaires internationaux et de leurs motivations à entrer et rester au HCR reste indispensable pour mieux saisir comment la construction de cette communauté d'appartenance s'articule avec des parcours individuels et des expériences sociales déjà marqués par un certain élitisme, communautarisme, ou cosmopolitisme. En entrouvrant quelques fenêtres sur les coulisses du HCR, il s'agissait surtout, dans cet article, de montrer comment les anthropologues ont encore à investir un vaste champ de recherche sur les processus d'action collective et les univers de sens qui sont à l'œuvre au sein des élites opérant à une échelle internationale.

\section{Références bibliographiques}

ABELES Marc (1995) Pour une anthropologie des institutions, L'Homme, 135, pp. 65-85.

AGAMBEN Giorgio (1995) Homo sacer : le pouvoir souverain et la vie nue, Le Seuil, Paris.

ANDERS Gerhard (2005) Good Governance as Technology - Toward an Ethnography of the Bretton

Woods Institutions, in D. Lewis and D. Mosse Eds., The Aid Effect: Giving and Governing in International Development, London, Pluto Press, pp. 37-60.

ATLANI-DUAULT Laëtitia (2005) Au bonheur des Autres. Anthropologie de l'aide humanitaire, Paris, Nanterre, Société d'Ethnologie.

BAGLA Lusin (2003) Sociologie des organisations, Paris, La Découverte.

BARÉ Jean-François Éd. (2006) Paroles d'experts : étude sur la pensée institutionnelle du développement, Paris, Karthala.

BELLIER Irène (1997) Une approche anthropologique de la culture des institutions, in Marc Abèles et H.P Jeudy Éds., Anthropologie du politique, Paris, Armand Colin, pp. 129-161.

BACKEWELL Oliver (2007) Researching refugees: Lessons from the past, current challenges and future directions, Refugee Quarterly Survey, vol. 26, issue 3, Oxford, pp. 6-14.

BOURDIEU Pierre (1988) La noblesse d'État. Grandes écoles et esprit de corps, Paris, Les éditions de Minuit.

BOURDIEU Pierre (1986) Les rites comme actes d'institution, in Pierre Centlivres et Jacques Hainard Dir., Les rites de passage aujourd'hui, Actes du colloque de Neuchâtel 1981, Lausanne, 
L'Âge d'homme, pp. 206-215.

CENTLIVRES Pierre (1986) Rites de passage : changement, opposition et contre-culture, in Pierre Centlivres et Jacques Hainard Dir., Les rites de passage aujourd'hui, Actes du colloque de Neuchâtel 1981, Lausanne, L'Âge d'homme, pp. 33-44.

CUCHE Denys (1988) La fabrication du « Gadz'arts », esprit de corps et inculcation culturelle chez les ingénieurs Arts et Métiers, Ethnologie française, XVIII, 1. pp. 42-54.

CZARNIAWSKA Barbara (1992) Exploring complex organizations. A cultural perspective, London, Sage.

DAUVIN Pascal et SIMEANT Johanna (2005) Le travail humanitaire. Les acteurs des ONG, du siège au terrain, Paris, Presses de sciences po.

DEMBOUR Marie Benedicte and KELLY Tobias (2007) Paths to International Justice: Social and Legal Perspectives, Cambridge, Cambridge University Press.

FOXEN P. (2009) Les réfugiés, in Laëtitia Atlani-Duault et Laurent Vidal, Anthropologie du développement et de l'aide humanitaire, Paris, Armand Colin, pp. 41-70.

FRESIA Marion (2007) Les réfugiés d'étude comme objet d'étude pour l'anthropologie, Refugee Quaterley Survey, 26 (3), pp. 100-118.

FRESIA Marion (2009) Protéger les réfugiés : normes et représentations, in Marion Fresia, Les Mauritaniens réfugiés au Sénégal, Paris, L'Harmattan, pp. 35-62.

FRESIA Marion (à paraître) Négocier des normes internationales sur la protection des réfugiés : regard sur les coulisses du comité exécutif du Haut Commissariat aux Réfugiés, Working Paper (2), Maison d'Analyse des Processus Sociaux, Neuchâtel.

GIOVALUCCHI François et OLIVIER DE SARDAN Jean-Pierre (à paraître), Planification, gestion et politique dans l'aide au développement : le cadre logique, outil et miroir des développeurs, Tiers Monde.

HAAS E.-B. (1990) When knowledge is power: three models of change in international organizations, Berkeley, University of California Press.

HARPER Richard (1997) Inside the IMF: An Ethnography of Documents, Technology and Organizational Action, London et San Diego, Academic Press.

HARREL-BOND Barbara (1986) Imposing Aid: emergency assistance to refugees, Oxford, Oxford University Press.

HERTZ E. (à paraître) Excessively Up at the International Labor Organization: Notes on "Note on the proceedings", TMITI/2007/10.

HUGUES Everett-C. (1996) Le regard sociologique. Essais choisis, Paris, Éditions de l'EHESS.

KOTT Sandrine (2008) Une « communauté épistémique » du social ? Experts de l'OIT et internationalisation des politiques sociales dans l'entre-deux-guerres, Genèses, 2 (71), pp. 26-46.

KUNDA Gideon (1992), Engineering culture, control and commitment in a high tech corporation, Philadelphia, University Press.

LAVIGNE-DELVILLE Philippe (2007) Prendre au sérieux les pratiques des développeurs : une étape nécessaire de l'analyse critique des interventions des ONG ?, Coopérer aujourd'hui, (53), Paris, GRET.

LOESCHER Gil (2001) The UNHCR and world politics: a perilous path, Oxford, Oxford University Press.

LOESCHER Gil, BETTS Alexander and MILLNER James (2008) The United Nations High Commissioner for Refugees (UNHCR). The politics and practice of refugee protection into the twenty-first century, London and New York, Routledge.

MALKKI Liisa (1995) Purity and Exile: violence, memory and national cosmology among Hutu refugees in Tanzania, Chicago, Chicago University Press.

MOSSE David (2005) Cultivating development: an ethnography of aid policy and practice, London, Pluto Press.

MULLER Birgit (2009) Comment rendre technique un débat politique. Controverses autour des biotechnologies agricoles au sein de la FAO, Tsantsa, 14, pp. 27-36. 
NADER Laura (1972) Up the anthropologist - perspectives gained from studying up, in Dell Hymes Ed., Reinventing Anthropology, New York, Random House.

STRAUSS A. et al. (1963) The hospital and its negotiated order, in E. Friedson Ed., The hospital in modern society, New York, Macmillan.

VALLUY Jérôme (2007) «Contribution à une sociologie politique du HCR : le cas des politiques européennes et du HCR au Maroc ", Asylon(s), 2, http://terra.rezo.net/article667.html, site consulté le 18 mai 2008.

VALLUY Jérôme (2009) Rejet des exilés : le grand retournement du droit d'asile, Broissieux, Paris, Éditions du Croquant.

VAN DAELE J. (2005) Engineering social peace: networks, ideas and the founding of international labor organization, International review of social history, 50, pp. 435-466.

WALKUP Mark (1997) Policy dysfunction in humanitarian organizations: the role of coping strategies, institutions and organizational culture, Journal of Refugee Studies, vol. 10 (1), pp. 37-60.

WIGLEY B. (2005) The state of UNHCR's organizational culture, Evaluation and policy analysis unit, UNHCR.

WRIGHT Susan Ed. (1994) Anthropology of organizations, London and New, York Routledge. 


\title{
Une élite transnationale : la fabrique d'une identité professionnelle chez les fonctionnaires du Haut Commissariat des Nations Unies aux Réfugiés
}

\author{
Marion FRESIA
}

\begin{abstract}
À partir de l'analyse de quelques catégories émic, omniprésentes dans les discours des fonctionnaires internationaux du Haut commissariat aux réfugiés, cet article s'intéresse à la manière dont les membres d'une élite transnationale mobilisent continuellement des cadres de référence communs, leur permettant de créer un certain " ordre » institutionnel au-delà de la pluralité de leurs origines nationales et de leur dispersion géographique. Il s'intéresse plus particulièrement aux éléments cognitifs, idéologiques, rituels ou pratiques qui participent à la fabrique de leur identité professionnelle : oscillant entre élitisme et communautarisme, travail diplomatique et action humanitaire, l'auteure montre comment celle-ci s'élabore avant tout dans une recherche de distinction par rapport aux autres, tout en se construisant quotidiennement à travers la défense d'une cause commune, des formes de socialisation fortement ritualisées, des repères spatio-temporels similaires, et le partage des mêmes frustrations vis-à-vis de sa profession.
\end{abstract}

\section{A Transnational Elite: The Making of a Professional Identity among the International Staff of the United Nations High Commissioner for Refugees}

\author{
Marion FRESIA
}

Based on an analysis of the discourse held by UNHCR staff on their work, this article explores how a transnational elite build common frames of references and produces institutional order, despite its conflicting interests, geographical dispersion and national heterogeneity. The author explores more particularly the ideological, cognitive and ritual elements which participate in the making of a common professional identity and sense of belonging among this elite. She highlights how this identity making process is based on a constant search for distinction vis-à-vis other humanitarian actors and on a structural tension between diplomatic work and humanitarian action, while taking shape on a daily basis through the sharing of common beliefs, social experiences and dilemma.

\section{Una élite transnacional: la fábrica de una identidad profesional de los funcionarios internacionales del HCR}

\section{Marion FRESIA}

Apoyándose sobre el análisis de algunas categorías emic omnipresentes en los discursos de los funcionarios del HCR cuando hablan de ellos mismos o de su organización, este artículo analiza como los miembros de una élite transnacional movilizan de manera continúa una referencia identitaria y cuadros de orientación comunes. Esto les permite de trabajar juntos y de crear un cierto «orden» institucional, más allá de la pluralidad de sus orígenes nacionales, de las tensiones que los dividen y de la dispersión espacial de los individuos. El artículo se interesa también a los elementos - cognitivos, ideológicos, rituales o prácticos - que participan a la fábrica de una identidad et de una comunidad profesional a escala internacional. 